International Journal of Biomedicine | June 2021 - Volume 11, Issue Suppl_1: Abstracts from the Third Russian International Conference "Cryo-electron microscopy 2021: achievements and prospects"

POSTER ABSTRACT PRESENTATIONS

SESSION TITLE: EM RESEARCH RELATED TO MEDICINE

DOI: 10.21103/IJBM.11.Suppl_1.P34

\title{
Abstract P-34: Cryo-EM Study of Submicrocapsules with a Shell of Nanoparticle Heteroaggregates and Polyelectrolyte Layers
}

\author{
$\underline{\text { Anastasiya Kostenko }}{ }^{1}$, Konstantin Palamarchuk $^{1}$, Yury Chesnokov ${ }^{1}$, \\ Konstantin Plokhikh $^{1}$, Tatyana Bukreeva ${ }^{1,2}$, Roman Kamyshinsky ${ }^{1,2}$ \\ ${ }^{I}$ National Research Centre "Kurchatov Institute," Moscow, Russia \\ ${ }^{2}$ Shubnikov Institute of Crystallography of FSRC "Crystallography and \\ Photonics" RAS, Moscow, Russia
}

Background: Currently, different approaches of active and passive targeted drug delivery are being developed. One of the most promising methods of targeted drug delivery is the use of capsules. For instance, colloidosomescapsules consisting of the shell formed by colloidal particles at the interface of the emulsion - can be used for targeted delivery of antitumor drugs or any other drugs in liquid form. Here we present results of cryo-EM study of submicrocapsules with the soybean oil core and with the shell consisting of $\mathrm{SiO} 2$ nanoparticles and detonation nanodiamonds (DNDs) stabilized with chitosan and alginate.

Methods: Cryo-electron tomography (Cryo-ET) was used to identify the morphological features of the submicrocapsules. Preliminary screening of samples and cryo-ET data collection were performed using Titan Krios cryo-EM (ThermoFisher Scientific, US) equipped with Falcon 2 direct electron detector. The restoration of the tomographic series was carried out using IMOD software. Eman2 was used for segmentation and UCSF Chimera was used for visualization of the 3D model. Submicron capsules were obtained by stabilizing oil droplets with a mixture of $\mathrm{SiO}_{2}$ nanoparticles and DNDs. To form a stable shell, an additional layer of silica particles and polyelectrolyte layers of alginate/chitosan were applied to the droplets of the dispersed phase of the emulsion by physical adsorption.

Results: Cryo-EM data showed the presence of submicrocapsules with a diameter in the range of $200-900 \mathrm{~nm}$. Although a significant fraction of submicrocapsules was found to be partially destroyed, results of cryo-ET 
study of intact capsules demonstrated that silicon dioxide nanoparticles form a net, while DNDs form clusters.

Conclusion: Here we demonstrate the results of the study of submicron capsules with a shell of silica nanoparticles and DNDs. It was found that a uniform distribution of DNDs is not a prerequisite for the creation of submicron capsules that contradicts the theoretical model.

\section{Key Words: submicrocapsules $\bullet$ cryo-EM $・$ cryo-ET}

This work was supported by the Russian Foundation for Basic Research (Grant No. 18-53-34007)

*Corresponding author: Anastasiya Kostenko. E-mail: nas-kostenko@mail.ru

International Journal of Biomedicine. 2021;11 Suppl 1: S26-27.

doi: 10.21103/IJBM.11.Suppl_1.P34

(C)2021 International Medical Research and Development Corporation 